\title{
Non-Competitive Fluorescence Polarization Immunoassay for Protein Determination
}

Mao Fukuyama $^{\dagger *}$, Ayano Nakamura ${ }^{\ddagger}$, Keine Nishiyama ${ }^{\ddagger}$, Ayuko Imai ${ }^{\sharp}$, Manabu Tokeshi ${ }^{\S}, K_{0 j i}$ Shigemura $^{*}$, and Akihide Hibara ${ }^{\dagger *}$.

${ }^{\dagger}$ Institute of Multidisciplinary Research for Advanced Materials, Tohoku University, Katahira 2-1-1, Aoba-ku, Sendai 980-8577, Japan

¥ Graduate School of Chemical Sciences and Engineering, Hokkaido University, Kita 13 Nishi 8, Kitaku, Sapporo 060-8628, Japan

${ }^{\S}$ Division of Applied Chemistry, Faculty of Engineering, Hokkaido University, Kita 13 Nishi 8, Kitaku, Sapporo 060-8628, Japan

\#Tianma Japan, Ltd., Shin-Kawasaki Mitsui Building West Tower 28F 1-1-2, Kashimada, Saiwai-ku, Kawasaki, Kanagawa 212-0058, Japan

Table of Contents.

1. Matrix effects on non-competitive fluorescence polarization immunoassay (FPIA) using VHH

2. Portable FPIA analyzer

3. Raw data of non-competitive FPIA using Fab and VHH obtained by the portable FPIA analyzer

4. The standard of ELISA assay for Human IgG 
1. Matrix effect on non-competitive fluorescence polarization immunoassay (FPIA) using VHH

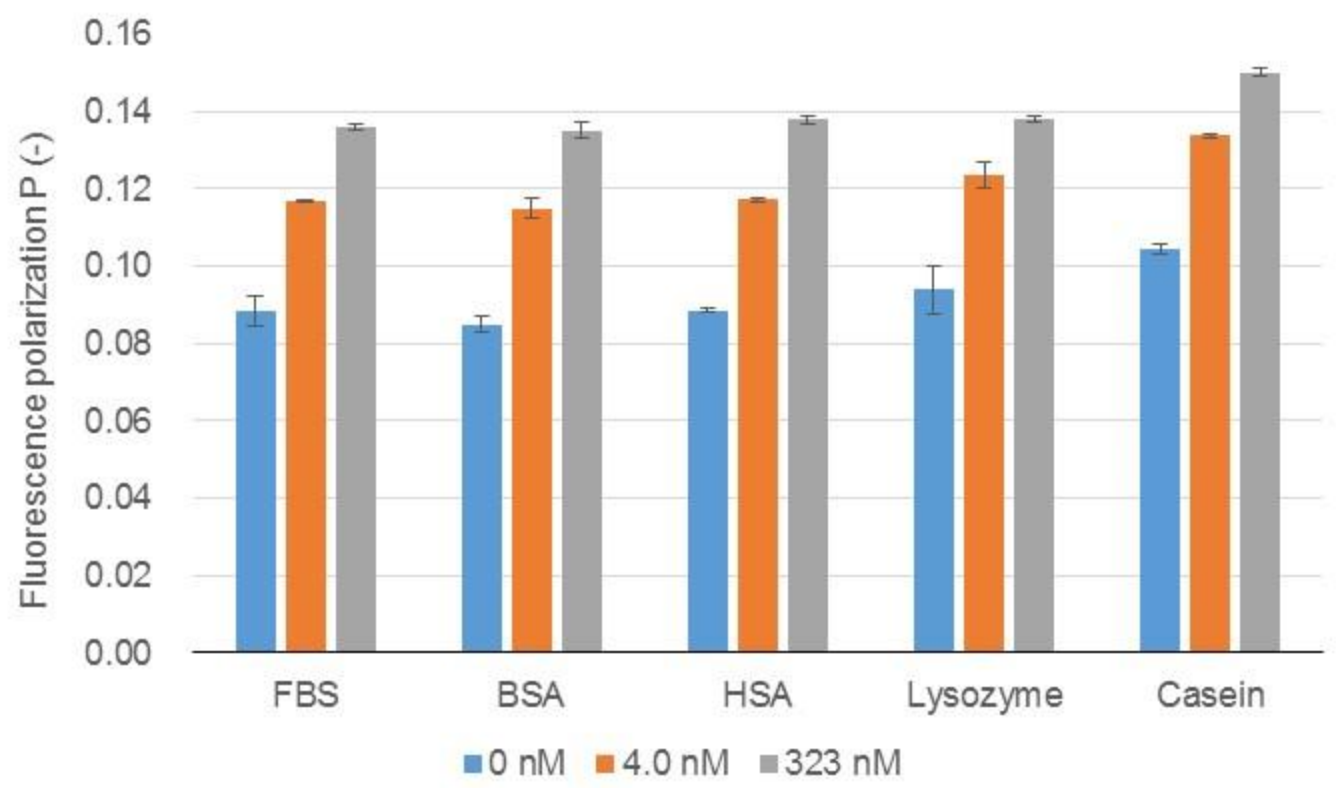

Figure S1. Non-competitive FPIA using VHH in difference protein solutions. Here, $0.1 \%$ solutions of fetal bovine serum (FBS), bovine serum albumin (BSA), human serum albumin (HSA), lysozyme and casein. $\mathrm{P}$ was measured with $0 \mathrm{nM}, 4.0 \mathrm{nM}$ and $323 \mathrm{nM}$ of rabbit IgG. 
2. Portable FPIA analyzer
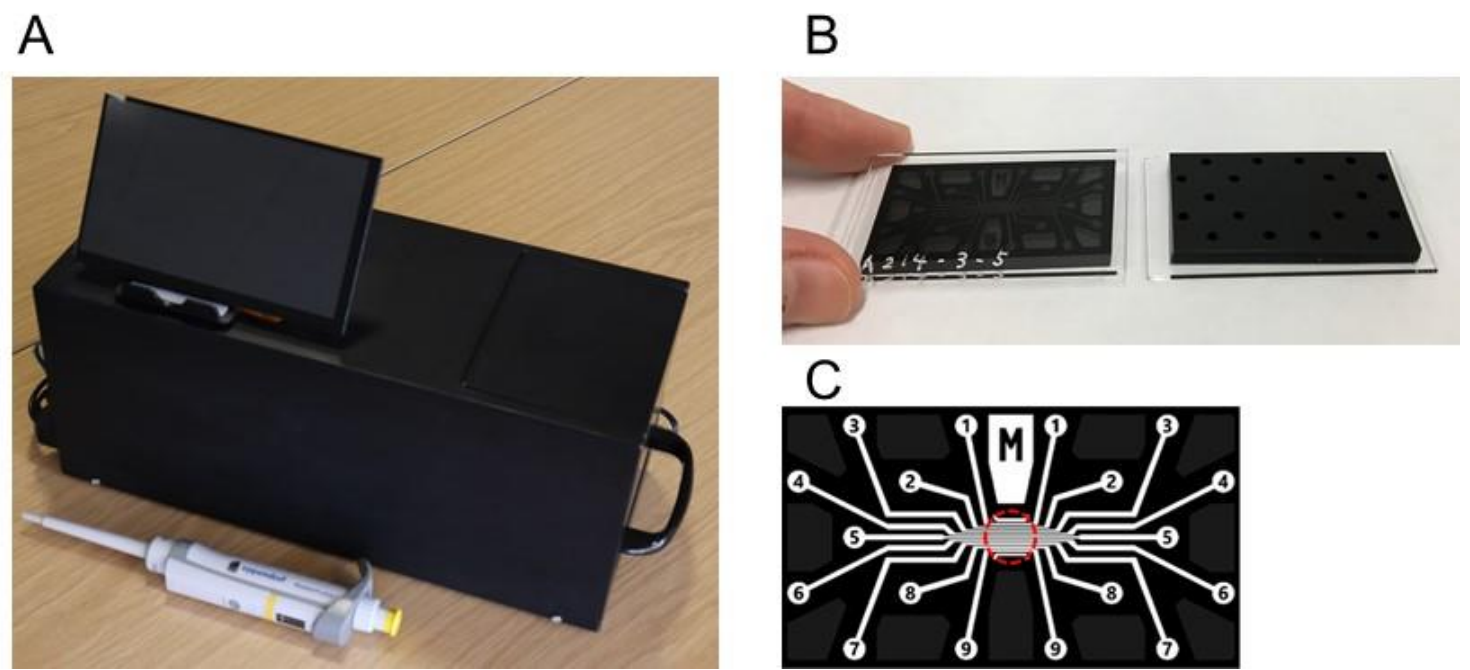

Figure S2. Portable FPIA analyzer. [1] (A) Photograph of the portable FPIA analyzer. (B) Photograph of PDMS microdevices. (C) Design of the microchannels. The red circle indicates the detection area. 
3. Raw data of non-competitive FPIA using Fab and VHH obtained by the portable FPIA analyzer
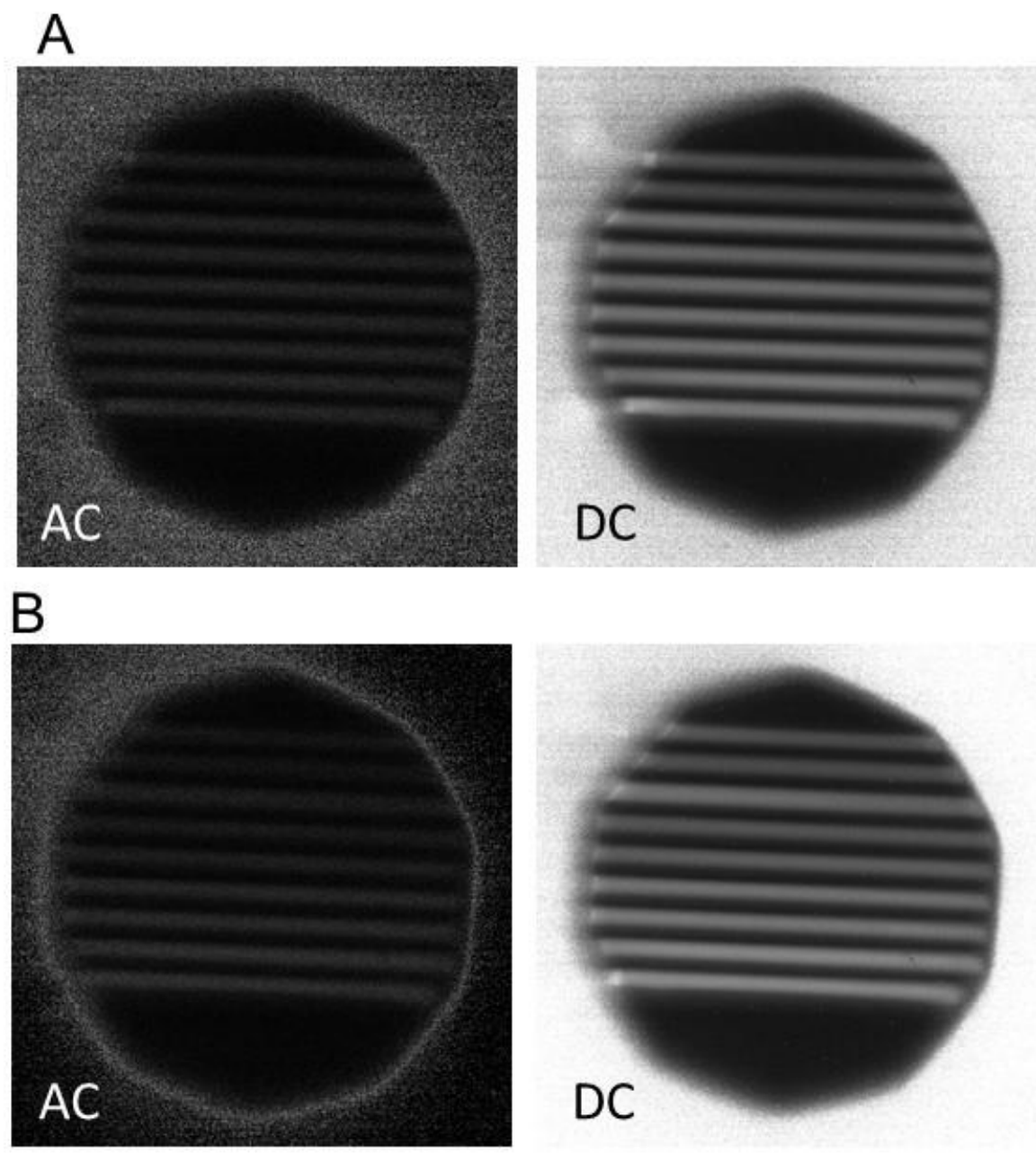

Figure S3. AC and DC images of non-competitive FPIA using Fab (A) and VHH (B) obtained by the portable FPIA analyzer. The concentration of the antigen increases from top to bottom. The $P$ value of each sample was calculated by analysing the grey value of each channel in these image using home-built image processing software[1,2]. 

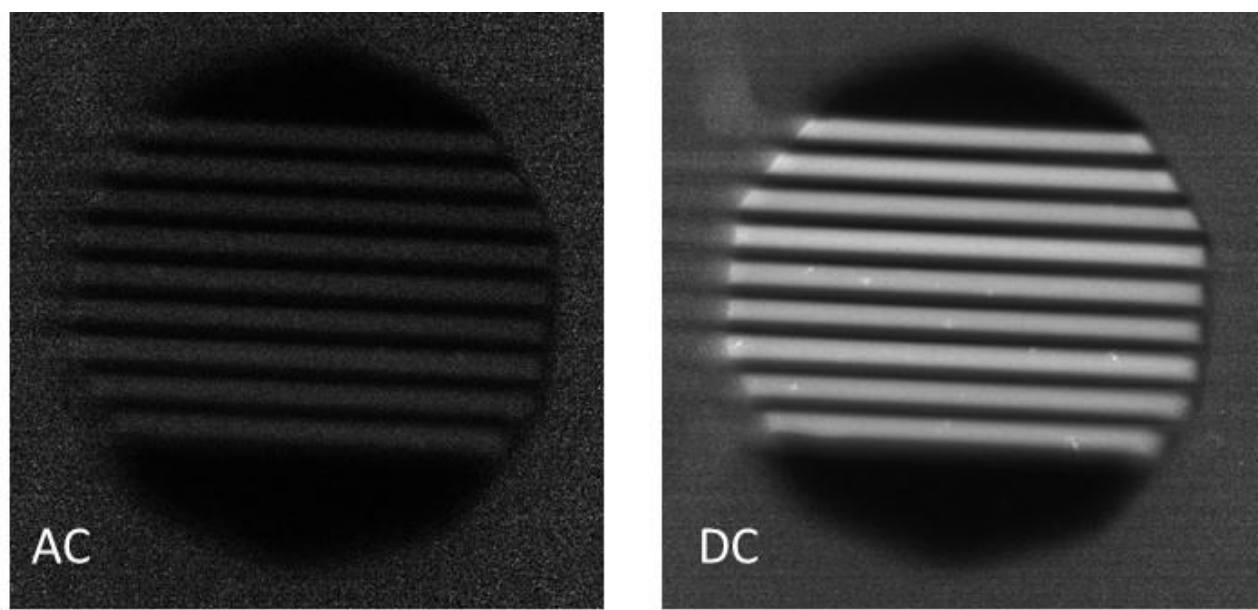

Figure S4. AC and DC images of rabbit IgG assay in the fetal bovine serum solution using noncompetitive FPIA obtained by the portable FPIA analyzer. The concentration of the antigen increases from top to bottom. The $P$ value of each sample was calculated by analysing the grey value of each channel in these image using home-built image processing software[1,2]. 
4. The standard curves of non-competitive FPIA using Fab and VHH and ELISA for Human IgG

a)

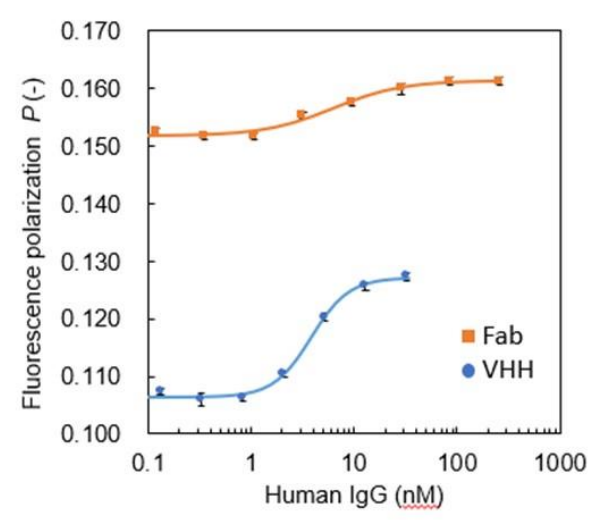

b)

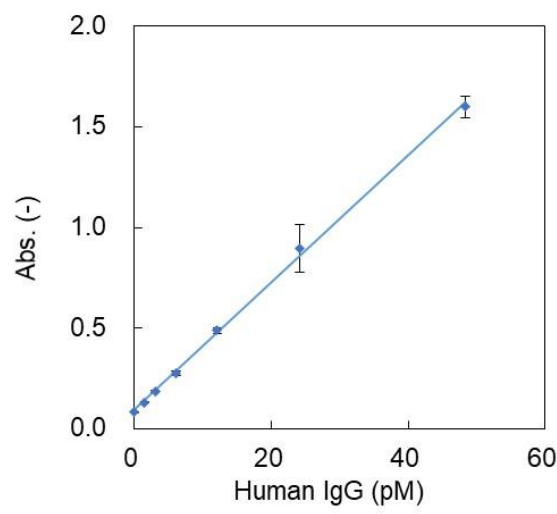

Figure S5. The standard curves for Human IgG quantification. (1) non-competitive FPIA using Fab and VHH. The solid lines indicate the sigmoidal curves fitted to the measurement results. (b) ELISA. The solid lines indicate the linear fitting.

\section{References}

[1] K. Nishiyama, Y. Takeda, M. Maeki, A. Ishida, H. Tani, K. Shigemura, et al., Rapid detection of anti-H5 avian influenza virus antibody by fluorescence polarization immunoassay using a portable fluorescence polarization analyzer, Sensors and Actuators B: Chemical, 316(2020) 128160.

[2] O. Wakao, Y. Fujii, M. Maeki, A. Ishida, H. Tani, A. Hibara, et al., Fluorescence Polarization Measurement System Using a Liquid Crystal Layer and an Image Sensor, Analytical Chemistry, 87(2015) 9647-52. 